\title{
Mathematical Quad Cross-Stub Stepped Impedance Resonator Application
}

\author{
Article Info \\ Page Number: $01-04$ \\ Publication Issue: \\ Vol 70 No. 1 (2021)
}

\author{
Viviana Lori \\ University of Ottawa \\ Canada
}

\author{
Article History \\ Article Received: 20 September 2020 \\ Revised: 15 October 2020 \\ Accepted: 02 December 2020 \\ Publication: 31 January 2021
}

\begin{abstract}
This simulation study involved the use of a QC-SSIR (quad cross-tub stepped impedance resonator) to examine the behavior and performance of the MW-BPF (multi-wideband band pass filter). To evaluate the performance of the proposed model, it was compared to the case of the conventional resonator. In the results, MW-BPF was found o exhibit a superior performance in terms of the ease of fabrication, good transmission coefficients, and a wider fractional bandwidth. For the filter structure analysis, this study incorporated the ABCD matrix, with the design of the MW-BPF also based on the FR4 microstrip. The experimental conditions and parameters were set in such a way that for the substrate, tan $\square=$ 0.0265 , thickness $=1.6 \mathrm{~mm}$, and $\square \mathrm{r}=4.4$, and thickness $\mathrm{h}=1.6 \mathrm{~mm}$. at $2.58 \mathrm{GHz}, 1.71 \mathrm{GHz}$, and $0.81 \mathrm{GHz}$, the proposed QC-SSIR-based MWBPF achieved transmission coefficients/fractional bandwidths of $1.93 \mathrm{~dB} / 13.9 \%, 1.49 \mathrm{~dB} / 18.7 \%$, and $0.60 \mathrm{~dB} / 49.3 \%$, respectively. Relative to the filter size reduction, the FQC-SSIR (folded quad cross-tub impedance resonator) was incorporated. The resultant observations indicated a possibility of BPF size reduction up to $46 \%$. Also, the proposed framework was found to yield (at 2.62, 1.80, and $0.82 \mathrm{GHz}$ ) $1.76 \mathrm{~dB} / 12.5 \%$, $1.21 \mathrm{~dB} / 17.7 \%$, and $0.57 \mathrm{~dB} / 49.6 \%$ transmission coefficient/fractional bandwidths, respectively. It is also worth indicating that the filter employed LTE2600, WCDMA1800, and GSM800.

Keywords: Cross-Stub, knowledge, mining, sentiment, tweet.
\end{abstract}

\section{Introduction}

In the design of multiband microwave filter, a significant role is played by microstrip filters, which are beneficial relative to low cost, reduced loss, miniaturization, and fabrication simplicity [1-6]. Some of the approaches that have gained increasing use in the design of multiband bypass filters include crossed open stubs, DGS resonator and stub-loaded resonator, stub-loaded quarter wavelength resonator, tri mode stub-loaded resonators, stubloaded resonators, loaded crossed resonators, cascaded multiband resonators, tri-section SIRs, and quarter-wavelength SIRs [7-10]. Indeed, in the design of the multi-band BPF, an emerging issue involves allowing various pass bands simultaneously while ensuring that the design freedom is not compromised. Another problem comes in terms of complex geometry and size increase. From recent scholarly studies, focus has been on the multi-band pass filter, with most of the findings demonstrating that the model exhibits a superior electrical performance $[2,4]$. Despite this promising outcome, however, there tends to be a BPF size increase while the bandwidth becomes narrower. For studies that have focused on 
miniaturized microstrip bandpass filters, specific forms on the focus have included the microstrip Y-shape bandpass filter, microstrip E-shape bandpass filter, and the rectangular dual spiral resonator (DSR) [22-24]. In the current investigation, focus is on the QC-SSIR microstrip model proposal, aimed at supporting the MW-BPF design.

\section{$2 \quad$ Methodology}

As mentioned earlier, the COS method, previously proposed in some studies $[3,4,6]$, was used in conjunction with the multi-band bandpass filter. Figure 1 demonstrates this approach. Equation 1 highlights the COS's ABCD matrix, demonstrating aspects of electrical length and the transmission line impedance $[6,16]$.

$\left[\begin{array}{ll}A & B \\ C & D\end{array}\right]=\left[\begin{array}{cc}\cos \theta_{1} & j Z_{1} \sin \theta_{1} \\ \frac{j \sin \theta_{1}}{Z_{1}} & \cos \theta_{1}\end{array}\right]\left[\begin{array}{cc}1 & 0 \\ \frac{j \sin \theta_{2}}{Z_{2}} & 1\end{array}\right]\left[\begin{array}{cc}1 & 0 \\ \frac{j \sin \theta_{3}}{Z_{3}} & 1\end{array}\right]\left[\begin{array}{cc}\cos \theta_{1} & j Z_{1} \sin \theta_{1} \\ \frac{j \sin \theta_{1}}{Z_{1}} & \cos \theta_{1}\end{array}\right]$

with $\theta_{0}$ is set to $\lambda / 4$ line or $90^{\circ}$, and $Z_{1}, Z_{2}$, and $Z_{3}$ can be found as follows [16]:

$$
\begin{aligned}
& Z_{1}=\frac{Z}{\tan \theta_{1}} \\
& \frac{\tan \theta_{2}}{Z_{2}}+\frac{\tan \theta_{3}}{Z_{3}}=\frac{\left(1-\tan ^{2} \theta_{1}\right)}{Z} \text { (3) }
\end{aligned}
$$

and the transmission zero created by [16]:

$$
\frac{f_{\theta_{2}}}{f_{0}}=\frac{\pi}{2 \theta_{2}}
$$

Of importance to note is that the COS approach, a conventional technique, gains much application to scenarios involving narrow bandwidths. Therefore, this study sought to achieve bandwidth expansion in which the QC-SSIR model was proposed for purposes of BPF generation. The QC-SSIR structure in relation to its electrical length and impedance of transmission is shown in Figure 2. To achieve the QC-SSIR's ABCD matrix, we have:

$\left[\begin{array}{ll}A & B \\ C & D\end{array}\right]=M_{1} M_{2} M_{34} M_{5} M_{2} M_{34} M_{5} M_{34} M_{2} M_{5} M_{34} M_{2} M_{1}$

with

$M_{1}=\left[\begin{array}{cc}\cos \theta_{1} & j Z_{1} \sin \theta_{1} \\ \frac{j \sin \theta_{1}}{Z_{1}} & \cos \theta_{1}\end{array}\right]$

$M_{2}=\left[\begin{array}{cc}1 & 0 \\ \frac{j \tan \theta_{2}}{Z_{2}} & 1\end{array}\right]$

$M_{5}=\left[\begin{array}{cc}\cos \theta_{5} & j Z_{5} \sin \theta_{5} \\ \frac{j \sin \theta_{5}}{Z_{5}} & \cos \theta_{5}\end{array}\right]$

For the case of $\mathrm{M}_{34}$, the step impedance resonator (SIR) structure determines its ABCD matrix. The resultant $\mathrm{ABCD}$ matrix comes in the form:

$M_{34}=\left[\begin{array}{cc}1 & 0 \\ Y_{S I R(34)} & 1\end{array}\right]$

Where:

$Y_{S I R(34)}=j Y_{3} \frac{Z_{3} \tan \theta_{4}+Z_{4} \tan \theta_{3}}{Z_{4}-Z_{3} \tan \theta_{3} \tan \theta_{4}}(10)$

Vol. 70 No. 1 (2021) 
When the same method is employed as that which has been proposed in some of the previous studies $[16,17]$, the $\lambda / 4$ line is used to replace the QC-SSIR. For the $\lambda / 4$ line, its ABCD matrix becomes:

$\left[\begin{array}{ll}A & B \\ C & D\end{array}\right]=\left[\begin{array}{cc}\cos \theta_{0} & j Z_{1} \sin \theta_{0} \\ \frac{j \sin \theta_{0}}{Z_{0}} & \cos \theta_{0}\end{array}\right]=\left[\begin{array}{cc}0 & j Z_{0} \\ j Y_{0} & 0\end{array}\right]$

When equations (11) and (5) are solved, we can obtain the electrical length and transmission line impedance values. In turn the $S_{12}$ and $S_{11}$ scattering parameters can be established through equations (13) and (12) $[16,17]$ in the form:

$$
\begin{aligned}
& S_{11}=\frac{A+\frac{B}{z_{0}}-C Z_{0}-D}{A+\frac{B}{z_{0}}+C Z_{0}+D} \\
& S_{21}=\frac{2}{A+\frac{B}{z_{0}}+C Z_{0}+D}
\end{aligned}
$$

On the microstrip, the fabrication of the MW-BPF was achieved via: $\tan \delta=0.0265$ and $\varepsilon \mathrm{r}=$ $4.4, \mathrm{~h}=1.6 \mathrm{~mm}$. Also, the QC-SSIR resonator was used to develop the filter - having considered a single layer substrate. With the QC-SSIR-based MW-BPF having its size set at $97 \mathrm{~mm}$ x $240 \mathrm{~mm}$, specific experimental conditions were summarized as follows:

$\mathrm{w}_{0}=3.5 \mathrm{~mm}, 1_{0}=25 \mathrm{~mm}, \mathrm{w}_{1}=1.5 \mathrm{~mm}, 1_{1}=1 \mathrm{~mm}, \mathrm{w}_{2}=1.5 \mathrm{~mm}, 1_{2}=1.5 \mathrm{~mm}, \mathrm{w}_{3}=2.5 \mathrm{~mm}$, $1_{3}=77 \mathrm{~mm}, \mathrm{w}_{4}=7.5 \mathrm{~mm}, 1_{4}=20 \mathrm{~mm}, \mathrm{w}_{5}=2.5 \mathrm{~mm}, 1_{5}=9 \mathrm{~mm}$

Regarding filter size reduction, the proposed approach entailed the folded QC-SSIR. To achieve the miniaturization process, $1_{3}$ 's length transmission line was rotated or folded to an extent that a length of $77 \mathrm{~mm}$ was achieved. For the first and fourth COS, two parts were obtained for $1_{3}$, which constitutes $36 \mathrm{~mm}$ and $41 \mathrm{~mm}$. for the case of the second and third COS, there was the division of $1_{3}$ to obtain four parts in the form of 5, 11, 36, and $25 \mathrm{~mm}$ (see Figure 4). For the folded QC-SSIR-based MW-BPF, the total size stood at $97 \mathrm{~mm} \times 130 \mathrm{~mm}$. at this stage, $46 \%$ was the reduction in the total size of MW-BPF.

\section{$3 \quad$ Results and Discussion}

Results for the transmission coefficients' extraction are demonstrated, as well as the reflection coefficients' extraction, having varied the aspect of $1_{2}$. Particularly, the latter variation was in the range of $0.5 \mathrm{~mm}$ to $4.5 \mathrm{~mm}$. from the results, it is evident that for $\mathrm{f}_{1}, \mathrm{f}_{2}$, and $\mathrm{f}_{3}$, constant transmission coefficients are still achieved. Furthermore, the results suggest that for changes in $l_{2}$, only $\mathrm{f}_{2}$ and $\mathrm{f}_{3}$ are affected. Even at $\mathrm{l}_{2}=4.5 \mathrm{~mm}$, it is also observed that for $f_{3}$, the reflection coefficients still rated below $-10 \mathrm{~dB}$. Findings reflect the transmission coefficient extraction, as well as the reflection coefficient extraction, having varied 15 . The variation in 15 is in the range of $3 \mathrm{~mm}$ and $12 \mathrm{~mm}$. results suggest that the values of the reflection coefficients affect the $1_{5}$ variation. However, the latter scenario is seen to pose a minimal impact on the shift in frequency. From Figure 6 (a), MW-BPF exhibits good isolation and also comes with three transmission zeros. The transmission zeros frequencies occur in 3.07, 2.16, and 1.2 GHz. The measured and simulated results for QC-SSIR-based MW-BPF are compared. Indeed, this MW-BPF framework incorporating QC-SSIR is seen to achieve $1.93 \mathrm{~dB} / 13.9 \%, 1.49 \mathrm{~dB} / 18.7 \%$, and $0.60 \mathrm{~dB} / 49.3 \%$, as the transmission coefficients/fractional bandwidth for $2.58,1.71$, and $0.81 \mathrm{GHz}$, respectively. The first frequency has $\lambda_{\mathrm{G}}$ as the wavelength and $\lambda_{G}^{2}=0.167$ or $97 \mathrm{~mm} \mathrm{x} 240 \mathrm{~mm}$ as the size of the filter. The proposed QC-SSIR sought to achieve the objective of filter size reduction. With a folded QC-SSIR incorporated in the MW-BPF, $\lambda_{G}^{2}=0.09$ or $97 \mathrm{~mm}$ x $129 \mathrm{~mm}$ was the size. 
$46 \%$ was the final size after BPF size reduction, with the first frequency's wavelength being $\lambda_{\mathrm{G}}$. in Figure $8(\mathrm{~b})$, the illustration demonstrates a comparative analysis of the outcomes between the measured and the simulated MW-BPF, having incorporated the folded SSIR. For the case of the FQC-SSIR, when MW-BPF was incorporated, the resultant structure or model achieved $1.76 \mathrm{~dB} / 12.5 \%, 1.21 \mathrm{~dB} / 17.7 \%$, and $0.57 \mathrm{~dB} / 49.6 \%$ as the transmission coefficients/fractional bandwidth for $2.62,1.80$, and $0.82 \mathrm{GHz}$, respectively. These findings are summarized in Table 1, which provides a comparison of the performance of different BPF structures. It is also notable that the findings demonstrate that when the QC-SSIR model is incorporated, it can be used towards the production of the multiband band-pass filter for applications such as LTE2600, WCDMA1800, and GSM800. Similarly, there is an observed good agreement between the measured and the simulated outcomes, suggesting that the proposed design is valid and highly reliable.

\section{Conclusion}

In summary, a QC-SSIR model was proposed in this study. the proposed microstrip structure was responsible for MW-BPF generation. Also, there was the application of the folded QCSSIR structure with the aim of reducing the size of the filter, reducing the size of MW-BFP to about $46 \%$. From the outcome evaluation perspective, there was good agreement between the measured and the simulated results.

\section{References}

[1] D. G. Rahn et al., A fully integrated multiband MIMO WLAN transceiver RFIC, IEEE Journal of SolidState Circuits 40 (2005), 1629-1641, doi: 10.1109/JSSC.2005.852419

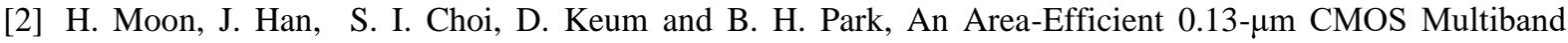
WCDMA/HSDPA Receiver, IEEE Transactions on Microwave Theory and Techniques 58 (2010), 14471455, doi: 10.1109/TMTT.2010.2042909

[3] T. Firmansyah, S. Praptodiyono, A. S. Pramudyo, C. Chairunissa, M. Alaydrus., Hepta-band bandpass filter based on folded cross-loaded stepped impedance resonator, Electronics Letters 53 (2017), 1119 - 1121, doi: 10.1049/el.2017.1121

[4] Firmansyah T, Praptodinoyo S, Wiryadinata R, et al, Dual-wideband band pass filter using folded crossstub stepped impedance resonator, Microw Opt Technol Lett 59 (2017), 2929-2934, doi: 10.1002/mop.30848

[5] R.-M. Weng and P.-Y. Hsiao, Double-layered quad-band bandpass filter for multi-band wireless systems, J Electromagn Waves Appl 23(2009), 2153-2161, doi: 10.1163/156939309790109324

[6] Wibisono G, Firmansyah T, Priambodo PS, et al. Multiband bandpass filter (BPF) based on folded dual crossed open stubs, Int J Tech 5(2014), 2-9, doi: 10.14716/ijtech.v5i1.151

[7] C. H. Lee, C. I. G. Hsu and H. K. Jhuang, Design of a New Tri-Band Microstrip BPF Using Combined Quarter-Wavelength SIRs, IEEE Microwave and Wireless Components Letters 16(2006), 594-596, doi: 10.1109/LMWC.2006.884902

[8] C. I. G. Hsu, C. H. Lee and Y. H. Hsieh, Tri-Band Bandpass Filter With Sharp Passband Skirts Designed Using Tri-Section SIRs, IEEE Microwave and Wireless Components Letters 18(2008), 19-21, doi: 10.1109/LMWC.2007.911976

[9] Yu-Cheng Chen, Yi-Huan Hsieh, Ching-Her Lee and Chung-I G. Hsu, Tri-band microstrip BPF design using tri-section SIRs, IEEE Antennas and Propagation Society International Symposium, Honolulu, 2007, 3113-3116, doi: 10.1109/APS.2007.4396195

[10] C. F. Chen, T. Y. Huang and R. B. Wu, Design of Dual- and Triple-Passband Filters Using Alternately Cascaded Multiband Resonators, IEEE Transactions on Microwave Theory and Techniques, 54 (2006), $3550-3558$ 